\title{
Transport characteristics of magnetite thin films grown onto GaAs substrates
}

\author{
S. M. Watts, ${ }^{a}$ K. Nakajima, S. van Dijken, and J. M. D. Coey \\ SFI Laboratory, Physics Department, Trinity College, Dublin 2, Ireland
}

(Presented on 9 January 2004)

\begin{abstract}
Magnetite thin films with a preferred (111) orientation have been deposited by reactive dc magnetron sputtering from a pure $\mathrm{Fe}$ target onto (100) GaAs substrates at $400{ }^{\circ} \mathrm{C}$. The films show a clear Verwey transition in both the magnetization and sheet resistance as functions of temperature. For films deposited onto semiconducting $n$-type GaAs substrates, we have obtained asymmetric current-voltage $(I-V)$ characteristics with a Schottky diodelike behavior in forward bias. Activation energy plots of the $I-V$ data as a function of temperature indicate a barrier height of $0.3-0.4 \mathrm{eV}$. This does not take into account the contribution from tunneling across the narrow depletion layer in these junctions, so should be considered a lower bound to the actual Schottky barrier height. Our work points to the potential integration of half-metallic magnetite with GaAs-based heterostructures for spin-electronic devices. (C) 2004 American Institute of Physics. [DOI: $10.1063 / 1.1652418]$
\end{abstract}

\section{INTRODUCTION}

Attempts to associate ferromagnetic metals with semiconductors in spin-electronic devices have met with limited success so far. One problem is the conductivity mismatch between metals and semiconductors that makes spin injection from the metal to the semiconductor difficult. ${ }^{1}$ One approach to the problem is to turn to more exotic materials, such as half-metallic ferromagnets, which include some Heusler alloys, perovskite manganites, $\mathrm{CrO}_{2}$, and $\mathrm{Fe}_{3} \mathrm{O}_{4} \cdot{ }^{2}$ The predicted half-metallicity of these materials means that the conduction electrons are completely spin polarized (at least at low temperature). The use of half-metals as spin injectors should circumvent many of the problems that plague conventional ferromagnetic metal/semiconductor contacts. But these are often hard to produce in thin-film multilayer structures without disrupting the stoichiometry necessary for optimal spin polarization. Furthermore, most methods for testing the degree of spin polarization in transport measurements require multilayer device fabrication. $\mathrm{CrO}_{2}$ is one such material that has been convincingly shown to be a completely spinpolarized source in a device geometry, ${ }^{3}$ albeit only at low temperatures.

The inverse spinel magnetite shows a high degree of spin polarization at room temperature in photoemission ${ }^{4}$ and exhibits large magnetoresistive effects in point contact measurements. ${ }^{5}$ Interesting results have been found for $\mathrm{Fe}_{3} \mathrm{O}_{4} /$ manganite trilayer junctions ${ }^{6}$ and in $\mathrm{Fe}_{3} \mathrm{O}_{4}$-based spin valves. ${ }^{7}$ The oxide is not truly a metal but is rather a polaronic conductor with a thermally activated conductivity at room temperature. This could be an advantage when mating with semiconductors, as the relative resistivity match can be tuned, especially near the Verwey transition at $120 \mathrm{~K}$ where the resistivity in bulk single crystals increases by two orders of magnitude. ${ }^{8}$ To our knowledge, there have been

${ }^{\text {a)} E l e c t r o n i c ~ m a i l: ~ w a t t s s @ t c d . i e ~}$ few attempts to grow magnetite on semiconductor substrates, and no reports of transport measurements between magnetite and semiconductors. We have deposited magnetite films onto GaAs substrates and assessed the film quality with x-ray diffraction, magnetization, and in-plane transport measurements. We also present current-voltage $(I-V)$ measurements across the magnetite/GaAs interface.

\section{EXPERIMENTAL METHODS}

The $\mathrm{Fe}_{3} \mathrm{O}_{4}$ films were deposited onto heated GaAs substrates by reactive dc sputtering of an $\mathrm{Fe}$ target, at a constant power of $52 \mathrm{~W}$ under partial pressures of $\mathrm{Ar}$ and $\mathrm{O}_{2}$ of 3 $\times 10^{-3}$ mbar and $4 \times 10^{-5}$ mbar, respectively. The substrates were heated to $400{ }^{\circ} \mathrm{C}$, a temperature at which we obtain high-quality epitaxial growth of $\mathrm{Fe}_{3} \mathrm{O}_{4}$ onto $\mathrm{MgO}$ substrates $^{9}$ that exhibit a resistance change of an order of magnitude at the Verwey temperature $T_{V}$ of $120 \mathrm{~K}$. On GaAs, the film growth is generally polycrystalline, ${ }^{10}$ but the

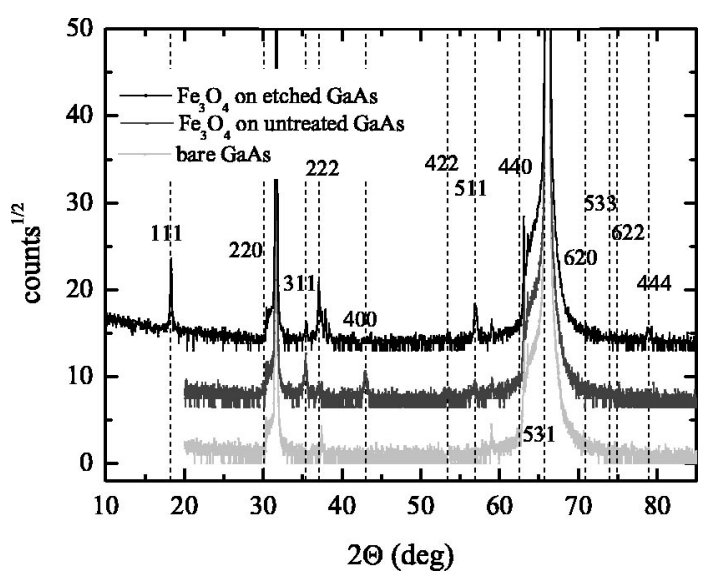

FIG. 1. X-ray diffraction curves for (from bottom) bare GaAs substrate, magnetite on an untreated GaAs substrate, and magnetite on a GaAs substrate pre-etched with an ammonia and hydrogen peroxide solution. 


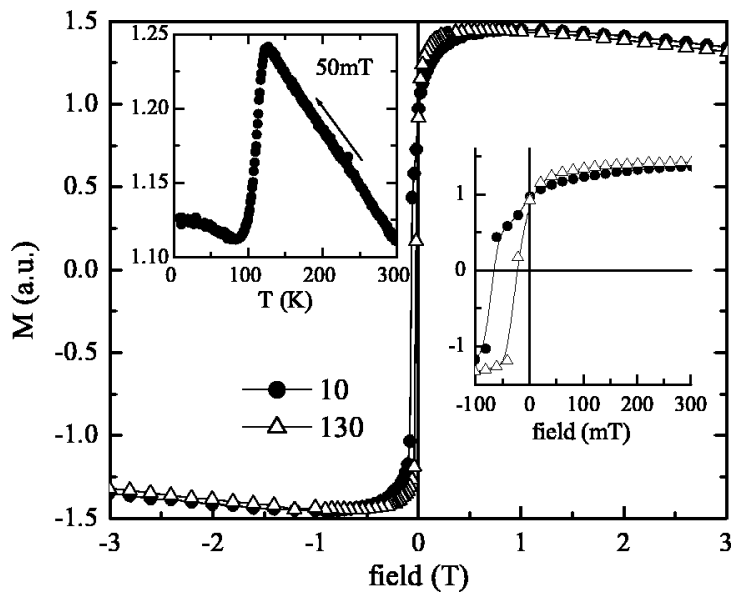

FIG. 2. Magnetization data showing the field dependence during a +5 to $-5 \mathrm{~T}$ sweep at temperatures 10 and $130 \mathrm{~K}$. The temperature dependence of the magnetization while cooling under a $50 \mathrm{mT}$ applied magnetic field is shown inset to the left-hand side. The inset to the right-hand side is an expanded view of the low-field magnetization data.

x-ray diffraction data in Fig. 1 show that when the substrates are etched in an ammonia and hydrogen peroxide solution to remove oxide surface layers prior to deposition, a preferential (111)-orientation develops out of plane. Similar results have been reported for sulfuric acid-based pre-etches. ${ }^{10}$

To examine transport properties through the film/ substrate interface, we used $n$-type GaAs substrates with a carrier concentration of $6-8 \times 10^{-17} \mathrm{~cm}^{-3}$. The back of the substrate was coated with In metal by soldering at $400^{\circ} \mathrm{C}$ prior to placing it into the vacuum chamber, where the contact was further annealed during the growth process at $400{ }^{\circ} \mathrm{C}$. The typical film thickness was $150 \mathrm{~nm}$, the area $2-4$ $\mathrm{mm}$ on a side. The magnetite surface was contacted with In solder at the corners. In-plane magnetotransport measurements were made in the van der Pauw geometry. $I-V$ measurements across the interface were made using one of the indium surface contacts.

\section{RESULTS}

Magnetization data taken in a Quantum Design superconducting quantum interference device magnetometer are shown in Fig. 2. The magnetization versus temperature data were recorded as the sample was cooled in a small in-plane applied magnetic field of $50 \mathrm{mT}$. The abrupt change in the magnetization near $120 \mathrm{~K}$ is due to the Verwey transition. The field dependence of the magnetization is shown for the temperatures $10 \mathrm{~K}$ and $130 \mathrm{~K}$ (just above the Verwey transition), for a single sweep from plus to minus $5 \mathrm{~T}$. The highfield slope is due to the diamagnetic contribution from the GaAs substrate and the In back contact. The right-hand side inset expands the low-field behavior, where it can be seen that the $10 \mathrm{~K}$ magnetization has a complicated switching behavior.

The $I-V$ curves taken while warming from $85 \mathrm{~K}$ are shown in Fig. 3. The data are asymmetric, with a diodelike behavior in forward bias typical of a Schottky barrier. The junctions exhibit a significant leakage current in reverse bias. The temperature dependence of the magnetite resistance

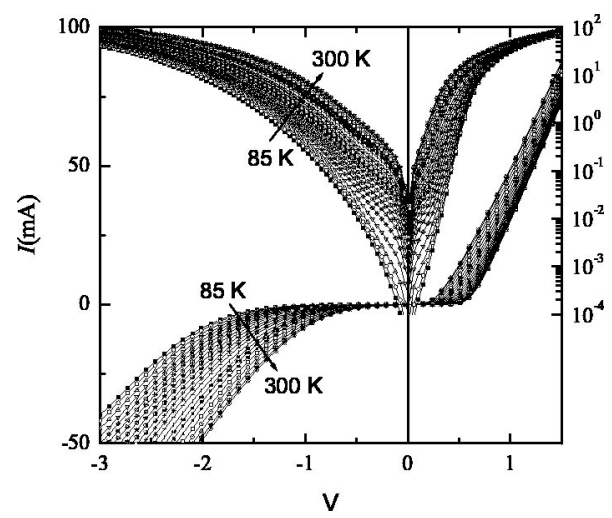

FIG. 3. $I-V$ traces at temperatures from 85 to $300 \mathrm{~K}$. The same data are shown on both a linear scale (left-hand side axis) and a semilog scale (righthand side axis, using the absolute value of the current).

shown in Fig. 4 was measured in the low-bias linear $I-V$ regime in order to avoid parallel conduction through the GaAs substrate. A deflection due to the Verwey transition can be observed around $110 \mathrm{~K} . R$ versus $T$ curves obtained from films grown onto $\mathrm{MgO}$ and $\mathrm{SrTiO}_{3}$ substrates are shown for comparison. The magnetoresistance (MR) was measured in the van der Pauw geometry at 90 and $120 \mathrm{~K}$, with magnetic field applied perpendicular to the film plane. The MR data, inset Fig. 4, shows the negative quasilinear dependence typical of magnetite, with a magnitude of $5 \%$ in $2.5 \mathrm{~T} 120 \mathrm{~K}$.

\section{DISCUSSION}

The x-ray diffraction, magnetization, and magnetotransport establish the quality of the magnetite films. The magnetization data show a clear, if somewhat broadened Verwey transition near $120 \mathrm{~K}$, consistent with bulk magnetite. The $R$ versus $T$ shows only a small inflection at $T_{V}$ which is similar to magnetite films grown at $300{ }^{\circ} \mathrm{C}$ on $\mathrm{SrTiO}_{3},{ }^{9}$ included for comparison in Fig. 4. The films have similar magnetotransport properties to those grown onto oxide substrates that are oriented out of plane, but are random in plane. ${ }^{11,12}$ The quality of in-plane transport measurements is reduced due to the intergranular disorder.

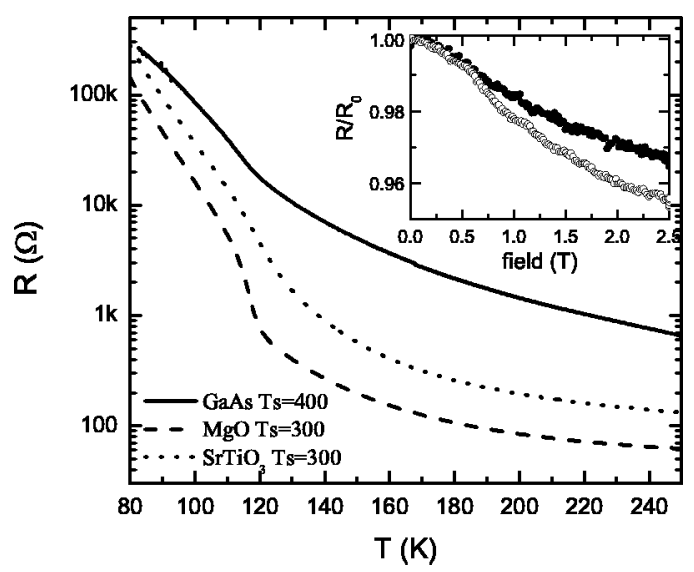

FIG. 4. Resistance vs temperature. Data are shown for magnetite films on $\mathrm{MgO}$ and $\mathrm{SrTiO}_{3}$ for comparison. The inset shows the $\mathrm{MR}$ at $90 \mathrm{~K}(\mathbf{O})$ and $120 \mathrm{~K}(\bigcirc)$. 


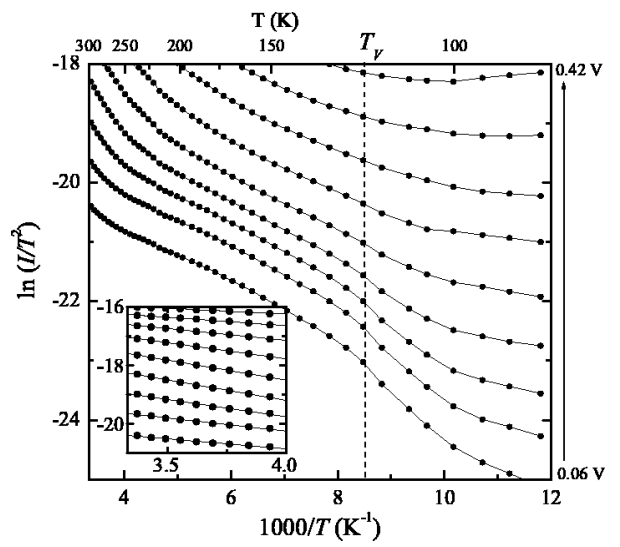

FIG. 5. Activation energy plot of constant voltage curves, based on Eq. (1). The inset shows linear fits to the data near room temperature for the same bias voltage range, 0.06 to $0.42 \mathrm{~V}$.

The $I-V$ characteristics indicate the presence of a Schottky barrier, but significant leakage current is evident in reverse bias. The carrier concentration of the GaAs substrates is high enough that both thermionic emission/ diffusion and tunneling processes should contribute at room temperature, the latter giving rise to some of the observed leakage, and tunneling should dominate at lower temperature.

In the thermionic emission/diffusion model, ${ }^{13}$ the barrier height may be obtained from an activation energy plot for which it is not necessary to specify the Richardson constant $A^{* *}$ or the junction area $A_{e}$. The slope of the data plotted as $\ln \left(I / T^{2}\right)$ versus $1 / T$ at a given forward bias $V$ yields the barrier height $\phi$ via the relation

$$
\ln \left(I / T^{2}\right)=\ln \left(A_{e} A^{* *}\right)-q(\phi-V) / k T .
$$

Fitting data in the vicinity of room temperature, it is found that $\phi$ is rather dependent on the bias voltage. Between 0.2 and $0.3 \mathrm{~V}, \phi$ ranges from 0.3 to $0.4 \mathrm{eV}$. However, the intercept does not return a reasonable value for $A_{e} A^{* *}$, due possibly to the influence of tunneling mechanisms as the temperature is decreased. Because of the tunneling contribution, the barrier height calculated using just the thermionic emission/diffusion model should be considered an underestimate, or lower limit to the actual Schottky barrier height.

It is interesting to examine the activation energy plot over the full range of temperature 85 to $300 \mathrm{~K}$, shown in Fig. 5 for various values of the forward bias. At low bias, there are three distinct temperature regimes: A high-temperature region of larger slope, a broad intermediate temperature re- gion $T_{V} \leqslant T \leqslant 250$ of smaller slope (leading to a barrier height of $\approx 0.14 \mathrm{~V}$ ), and a faint kink near $T_{V}$ where the slope appears to increase again. The intermediate regime is where transport is dominated by tunneling over thermionic emission. At $T_{V}$, the magnetite undergoes a transition that changes the transport mechanism from activated to variable range hopping. ${ }^{8}$ The data suggest that this reduces the tunneling probability and leads to an increase in the effective barrier height at $T \leqslant T_{V}$.

In conclusion, magnetite films have been grown onto (100) GaAs and show a preferred (111) out-of-plane orientation, a clear Verwey transition in the magnetization, and inplane magnetotransport that is similar in character to magnetite films grown onto $\mathrm{SrTiO}_{3}$ and $\mathrm{Al}_{2} \mathrm{O}_{3}$. Transport across the $\mathrm{Fe}_{3} \mathrm{O}_{4} / \mathrm{GaAs}$ interface at room temperature is due to both thermionic emission/diffusion across a Schottky barrier and tunneling through the narrow depletion layer for these highly doped substrates. At lower temperatures, tunneling is dominant but with a change in the tunneling probability at $T_{V}$. Considering the intrinsic high spin polarization and resistance tunability of magnetite, the work presented here points to the possibility of a tunable test system for spin injection into GaAs-based heterostructures.

\section{ACKNOWLEDGMENT}

This work was part of the CINSE project supported by Science Foundation Ireland. It was also supported by the EU Multimetox network, G5RT-CT-1999-05001. One of the authors (S. W.) would like to acknowledge the assistance of Plamen Stamenov in the experiments and the discussion.

${ }^{1}$ G. Schmidt, D. Ferrand, L. W. Molenkamp, A. T. Filip, and B. J. van Wees, Phys. Rev. B 62, R4790 (2000).

${ }^{2}$ J. M. D. Coey and M. Venkatesan, J. Appl. Phys. 91, 8345 (2002).

${ }^{3}$ J. S. Parker, S. M. Watts, P. G. Ivanov, and P. Xiong, Phys. Rev. Lett. 88, 196601 (2002).

${ }^{4}$ Yu. S. Dedkov, U. Rüdiger, and G. Güntherodt, Phys. Rev. B 65, 064417 (2002).

${ }^{5}$ J. J. Versluijs, M. A. Bari, and J. M. D. Coey, Phys. Rev. Lett. 87, 026601 (2001).

${ }^{6}$ G. Hu and Y. Suzuki, Phys. Rev. Lett. 89, 276601 (2002).

${ }^{7}$ S. van Dijken, X. Fain, K. Nakajima, S. M. Watts, and J. M. D. Coey (unpublished).

${ }^{8}$ F. Walz, J. Phys.: Condens. Matter 14, R285 (2002).

${ }^{9}$ S. M. Watts, S. van Dijken, and J. M. D. Coey (unpublished).

${ }^{10}$ R. J. Kennedy and P. A. Stampe, J. Phys. D 32, 16 (1999).

${ }^{11}$ G. Q. Gong, A. Gupta, Gang Xiao, W. Qian, and V. P. Dravid, Phys. Rev. B 56, 5096 (1997).

${ }^{12}$ S. B. Ogale, K. Ghosh, R. P. Sharma, R. L. Greene, R. Ramesh, and T. Venkatesan, Phys. Rev. B 57, 7823 (1998).

${ }^{13}$ S. M. Sze, Physics of Semiconductor Devices, 2nd ed. (Wiley, New York, 1981). 